\title{
ASYMPTOTIC EXPANSIONS FOR SOME SEMIPARAMETRIC PROGRAM EVALUATION ESTIMATORS ${ }^{*}$
}

\author{
by \\ Hidehiko Ichimura \\ University College, London \\ Oliver Linton \\ London School of Economics and Political Science
}

Contents:

Abstract

1. Introduction

2. The Model and the Estimator

3. Main Results

4. Some Numerical Results

5. Conclusions

Appendix

References

Figures

Discussion Paper

No.EM/03/451

May 2003
The Suntory Centre

Suntory and Toyota International Centres for Economics and Related Disciplines London School of Economics and Political Science Houghton Street London WC2A 2AE Tel.: 02079556698

\footnotetext{
*We would like to thank Tom Rothenberg, two referees, and seminar participants for helpful comments, and David Jacho-Chavez for research assistance. We are grateful to the National Science Foundation, to CEMMAP, and to the Economic and Social Research Council for financial support.
} 


\begin{abstract}
We investigate the performance of a class of semiparametric estimators of the treatment effect via asymptotic expansions. We derive approximations to the first two moments of the estimator that are valid to 'second order'. We use these approximations to define a method of bandwidth selection. We also propose a degrees- of-freedom like bias correction that improves the second order properties of the estimator but without requiring estimation of higher order derivatives of the unknown propensity score. We provide some numerical calibrations of the results.
\end{abstract}

Keywords: bandwidth selection; kernel estimation; program evaluation; semiparametric estimation; treatment effect.

JEL No.: C14

(C) by the authors. All rights reserved. Short sections of text, not to exceed two paragraphs, may be quoted without special permission provided that full credit, including (C) notice, is given to the source.

Contact address:

Professor Oliver Linton, Department of Economics, London School of Economics and Political Science, Houghton Street, London WC2A 2AE, UK. Email:

o.linton@Ise.ac.uk 


\section{Introduction}

In a series of classic papers Tom [Rothenberg $(1984 \mathrm{abc}, 1988)]$ introduced Edgeworth expansions to a broad audience. His treatment of the generalized least squares estimator (1984b) in particular was immensely influential because it dealt with an estimator of central importance and the analysis was both deep and precise, but comprehensible. This is in contrast with some of the more frenzied publications about Edgeworth expansions that had hitherto appeared in econometrics journals. The use of Basu's theorem in that paper to establish the independence of the correction terms from the leading term is a well-known example of his elegant work. The review paper (1984a) was also very influential and highly cited.

It is our purpose here to present asymptotic expansions for a class of semiparametric estimators used in the program evaluation literature. We have argued elsewhere, Linton $(1991,1995)$ and Heckman, Ichimura, Smith and Todd (1998), that the first-order asymptotics of semiparametric procedures can be misleading and unhelpful. The limiting variance matrix of the semiparametric procedure $\Sigma$ does not depend on the specific details of how the nonparametric function estimator $\widehat{g}$ is constructed, and thus sheds no light on how to implement this important part of the procedure. Specifically, bandwidth choice cannot be addressed by using the first-order theory alone. Also, the relative merits of alternative first-order equivalent implementations, e.g., one-step procedures, cannot be determined by the first-order theory alone. Finally, to show when bootstrap methods can provide asymptotic refinements for asymptotically pivotal statistics requires some knowledge of higher-order properties - see Horowitz (1995). This motivates the study of higher-order expansions. Carroll \& Härdle (1989) was to our knowledge the first published paper that developed second-order mean squared error expansions for a semiparametric, i.e., smoothing-based but root-n consistent, procedure, in the context of a heteroskedastic linear regression. Härdle, Hart, Marron, \& Tsybakov (1992) developed expansions for scalar average derivatives which was extended to the multivariate case, actually only the simpler situation of density-weighted average derivatives, by Härdle \& Tsybakov (1993); these papers used the expansions to develop automatic bandwidth selection routines. This work was extended to the slightly more general case of density-weighted averages by Powell \& Stoker (1996). In the second author's PhD thesis [Linton (1991)], written under Tom's supervision, the second author developed expansions for a variety of semiparametric regression models including the partially linear model and the heteroskedastic linear regression model; some of this work was later published in Linton $(1995,1996 \mathrm{a})$. The Linton (1995) paper also provided some results on the optimality of the bandwidth selection procedures proposed therein. Xiao \& Phillips (1996) worked 
out the same approximations for a time series regression model with serial correlation of unknown form; Xiao \& Linton (2001) give the analysis for Bickel's (1982) adaptive estimator in the linear regression model; Linton \& Xiao (1997) works out the approximations for the nonlinear least squares and profile likelihood estimators in a semiparametric binary choice model. Nishiyama \& Robinson (2000) proved the validity of an Edgeworth approximation to the distribution of the density weighted average derivative estimator. Linton (2001) derived an Edgeworth approximation to the distribution of the standardized estimator and a Wald statistic in a semiparametric instrumental variables model.

In this paper, we develop asymptotic expansions for an estimator of the treatment effect recently proposed in Hirano, Imbens, \& Ridder (2000), henceforth HIR. Propensity Score matching is a nonexperimental method for estimating the average effect of social programs. ${ }^{1}$ The method compares average outcomes of participants and nonparticipants conditioning on the propensity score value. When averaged over the propensity score, the average measures the average impact of a program if the conditioning on the observable variables makes the choice of the program conditionally mean independent from the potential outcomes. This methodology has received much attention recently in econometrics. While the method used often in practice uses the nearest match in either regressors or estimated propensity score to compare the treatment and the comparison groups, the asymptotic distribution theory for these methods have not been developed. The asymptotic distribution theory has been developed by Heckman, Ichimura \& Todd (1998) for the kernel based matching method. HIR considers reweighting estimator that estimates the treatment effect as well. Both methods require choosing smoothing parameters but optimal methods to choosing the smoothing parameter have not been discussed. In this paper we consider optimal bandwidth selection for the reweighting estimator.

\section{The Model and Estimator}

We investigate a class of estimators for the treatment effect, studied by HIR. Let $Y_{1}$ and $Y_{0}$ denote potential outcome for an individual with and without 'the treatment'. Define

$$
Y=Y_{1} \cdot T+Y_{0} \cdot(1-T)
$$

where $T$ is an indicator variable denoting the presence of treatment, i.e.,

$$
T= \begin{cases}1 & \text { if treated } \\ 0 & \text { if untreated }\end{cases}
$$

\footnotetext{
${ }^{1}$ See Cochran (1968), Rosenbaum \& Rubin (1983), and Heckman, Ichimura, \& Todd (1998).
} 
Let $X$ be a vector of covariates or pre-treatment variables. Actually, for convenience we will take $X$ to be a scalar and to have a continuous density $f$ bounded away from zero on its compact support. We will also assume that $Y$ possesses many finite moments. We are interested in the average treatment effect parameter

$$
\tau_{0}=E\left(Y_{1}\right)-E\left(Y_{0}\right) .
$$

We shall assume the following identifying conditions:

$$
\begin{aligned}
E\left[Y_{1} \mid X, T\right. & =1]=E\left[Y_{1} \mid X, T=0\right] \\
E\left[Y_{0} \mid X, T\right. & =1]=E\left[Y_{0} \mid X, T=0\right] \\
0 & <p(X)<1
\end{aligned}
$$

with probability one in $X$, where

$$
p(x)=\operatorname{Pr}[T=1 \mid X=x]=E(T \mid X=x)
$$

is the propensity score. The first two assumptions are that treatment and potential outcome are mean independent given covariates; the final assumption is that there are at least some unobserved influences on the probability of receiving the treatment. See Rosenbaum and Rubin (1983) and Heckman et al. (1998). Clearly under these assumptions $E\left[Y_{1} \mid X=x, T=1\right]=E\left[Y_{1} \mid X=x\right]=m_{1}(x)$ and $E\left[Y_{0} \mid X, T=0\right]=E\left[Y_{0} \mid X=x\right]=m_{0}(x)$. Furthermore, the following observable regressions are related to the unobservable regressions:

$$
\begin{aligned}
& g_{1}(x) \equiv E[Y \cdot T \mid X=x]=m_{1}(x) \cdot p(x), \text { and } \\
& g_{0}(x) \equiv E[Y \cdot(1-T) \mid X=x]=m_{0}(x) \cdot(1-p(x)) .
\end{aligned}
$$

It now follows that the average treatment effect parameter $\tau_{0}$ satisfies

$$
\begin{aligned}
\tau_{0} & =E\left(Y_{1}\right)-E\left(Y_{0}\right)=E\left[m_{1}(X)\right]-E\left[m_{0}(X)\right] \\
& =E\left[\frac{g_{1}(X)}{p(X)}\right]-E\left[\frac{g_{0}(X)}{1-p(X)}\right]=E\left[\frac{E(Y \cdot T \mid X)}{p(X)}\right]-E\left[\frac{E(Y \cdot(1-T) \mid X)}{1-p(X)}\right] \\
& =E\left[\frac{Y \cdot T}{p(X)}\right]-E\left[\frac{Y \cdot(1-T)}{1-p(X)}\right],
\end{aligned}
$$

where the last line follows from the law of iterated expectations. The last line is the relation that HIR use to suggest an estimator. Suppose now that we observe a sample $\left\{Z_{i}, i=1, \ldots, n\right\}$, where $Z_{i}=\left(Y_{i}, T_{i}, X_{i}\right)$. The HIR estimator is

$$
\widehat{\tau}=\frac{1}{n} \sum_{i=1}^{n}\left[\frac{Y_{i} \cdot T_{i}}{\widehat{p}\left(X_{i}\right)}-\frac{Y_{i} \cdot\left(1-T_{i}\right)}{1-\widehat{p}\left(X_{i}\right)}\right]
$$


where $\widehat{p}\left(X_{i}\right)$ was a nonparametric estimate of $p\left(X_{i}\right)$, in fact they chose series estimates.

We allow a slightly greater degree of generality; in particular, we consider the estimator $\widehat{\tau}$ of $\tau_{0}$ to be any sequence that solves

$$
\frac{1}{\sqrt{n}} \sum_{i=1}^{n} \Psi\left(Z_{i}, \tau, \widehat{p}\left(X_{i}\right)\right)=o_{p}\left(n^{-5 / 4}\right)
$$

where

$$
\Psi\left(Z_{i}, \tau, \widehat{p}\left(X_{i}\right)\right)=\frac{Y_{i} \cdot T_{i}}{\widehat{p}\left(X_{i}\right)}-\frac{Y_{i} \cdot\left(1-T_{i}\right)}{1-\widehat{p}\left(X_{i}\right)}-\tau
$$

and

$$
\widehat{p}\left(X_{i}\right)=\sum_{j=1}^{n} w_{i j} T_{j}
$$

where $w_{i j}$ are smoothing weights that only depend on the covariates $X_{1}, \ldots, X_{n}{ }^{2}$ As we mentioned earlier, HIR used series estimates. The bias correction method we propose below can also be applied to series estimates and indeed to any linear smoother, but detailed discussion of smoothing bias terms requires that we use kernel or local polynomial estimators. We will also adopt the leave-oneout paradigm that is used in many semiparametric estimates. To be specific we let the parameter vector $\left(\widehat{\alpha}_{0}\left(X_{i}\right), \widehat{\alpha}_{1}\left(X_{i}\right)\right)$ minimize the criterion function

$$
\sum_{j \neq i} K\left(\frac{X_{j}-X_{i}}{h}\right)\left\{T_{j}-\alpha_{0}+\alpha_{1}\left(X_{j}-X_{i}\right)\right\}^{2}
$$

with respect to $\left(\alpha_{0}, \alpha_{1}\right)$, where $K$ is a differentiable probability density function symmetric about zero with support $[-1,1]$, while $h=h(n)$ is a positive bandwidth sequence. Then let $\widehat{p}\left(X_{i}\right)=\widehat{\alpha}_{0}\left(X_{i}\right)$ and let $w_{i j}$ be the corresponding smoothing weights. We have taken the fixed bandwidth leave-one-out local linear kernel smoother as our estimator of the regression function. This estimator is preferable to the local constant kernel estimator because of its superior bias properties both at interior and boundary regions, see Fan and Gijbels (1996).

\section{Main Results}

HIR showed that the standardized estimator $\mathcal{T}=\sqrt{n}\left(\widehat{\tau}-\tau_{0}\right)$ satisfies

$$
\mathcal{T}=\frac{1}{\sqrt{n}} \sum_{i=1}^{n} \rho_{i}+o_{p}(1)=\mathcal{T}_{0}+o_{p}(1)
$$

${ }^{2}$ The precise magnitude of the error of (1) is sufficient for both of our higher order expansions in Theorems 1 and 2 below. It is certainly much smaller than is needed for root-n consistency. 
where $\rho_{i}=\Psi\left(Z_{i} ; \tau_{0}, p\left(X_{i}\right)\right)+s_{p}\left(X_{i}\right) \varepsilon_{i}$, where $\varepsilon_{j}=T_{j}-p\left(X_{j}\right)$ and

$$
s_{p}(x)=E\left[\Psi_{p}\left(Z_{i} ; \tau_{0}, p\left(X_{i}\right)\right) \mid X_{i}=x\right]=-\left[\frac{m_{1}(x)}{p(x)}+\frac{m_{0}(x)}{1-p(x)}\right] .
$$

Here, the derivatives of $\Psi$ with respect to $p$ are denoted by $\Psi_{p}, \Psi_{p p}$ etc. Therefore, $\mathcal{T}$ is asymptotically normal with finite variance

$$
v_{0}=E\left[\left(\Psi\left(Z_{i} ; \tau_{0}, p\left(X_{i}\right)\right)+s_{p}\left(X_{i}\right) \varepsilon_{i}\right)^{2}\right]
$$

In fact, they rewrote the asymptotic variance in the more interpretable form

$$
v_{0}=\operatorname{var}\left[E\left(Y_{1}-Y_{0} \mid X\right)\right]+E\left[\frac{\operatorname{var}\left(Y_{1} \mid X\right)}{p(X)}\right]+E\left[\frac{\operatorname{var}\left(Y_{0} \mid X\right)}{1-p(X)}\right] .
$$

They also established that this estimator is semiparametrically efficient, i.e., it has the smallest asymptotic variance amongst the class of all feasible estimators.

We are interested in the higher order properties of their estimator. We derive a stochastic expansion for $\mathcal{T}$ by Taylor expanding $\Psi\left(Z_{i}, \tau, \widehat{p}\left(X_{i}\right)\right)$ around $\Psi\left(Z_{i}, \tau, p\left(X_{i}\right)\right)$, thereby obtaining the representation

$$
\mathcal{T}=\mathcal{T}_{0}+\mathcal{T}_{1}+R=\mathcal{T}^{*}+R
$$

where the leading term $\mathcal{T}_{0}$ is as defined in (4), $\mathcal{T}_{1}$ contains the 'second order' terms, while $R$ is a remainder term that is of smaller order in probability. To be specific, we show that $R=o_{p}\left(n^{-\alpha}\right)$ in probability for some $\alpha>0$, where $\alpha$ is determined by the order of magnitude of the bandwidth and of course by how many terms in the Taylor expansion we retain. The magnitude $o_{p}\left(n^{-\alpha}\right)$ is determined to ensure that our results in Theorems 1 and 2 below are sensible. The random variable $\mathcal{T}^{*}$ has finite moments to various orders and indeed it is a linear combination of certain U-statistics. We shall calculate the moments of $\mathcal{T}^{*}$ and interpret them as if they were the moments of $\mathcal{T}$. This methodology has a long tradition of application in econometrics following Nagar (1959). ${ }^{3}$ The two largest [in probability] second order terms in $\mathcal{T}_{1}$ are both non-zero mean and are

$$
O_{p}\left(h^{2} \sqrt{n}\right)+O_{p}\left(n^{-1 / 2} h^{-1}\right) .
$$

\footnotetext{
${ }^{3}$ When $\sup _{n} E\left[\mathcal{T}^{2}\right]<\infty$, we might reasonably expect that $E\left[\mathcal{T}^{2}\right]=E\left[\mathcal{T}^{* 2}\right]+o\left(n^{-\alpha}\right)$, but see Srinavasan $(1970)$ for a cautionary tale in this regard. In any case, our $\mathcal{T}$ does not necessarily have uniformly bounded moments. Therefore, some additional justification for examining the moments of the truncated statistic must be given. With some additional work and regularity conditions it is possible to establish the stronger regularity that $\mathcal{T}$ and $\mathcal{T}^{*}$ have the same distribution to order $n^{-\alpha}$, which requires some restrictions on the tails of $R$, see the discussion in Rothenberg (1984a). In this case our moment approximations can be interpreted as the moments of the approximating distribution.
} 
In $\mathcal{T}_{1}$ there are also mean zero random variables of order $h^{2}$ and order $n^{-1 / 2} h^{-1 / 2}$. However, according to the criterion of mean squared error, these stochastic terms are dominated by the bias terms, and the optimal thing to do is to minimize the size of (7) by choosing $h$ appropriately. The optimal bandwidth is therefore of order $h \asymp n^{-1 / 3}$, in which case both terms in (7) are the same magnitude, and indeed are both of order $n^{-1 / 6}$. Thus, the second order terms are very large and are mostly bias related. This suggests that the usual (first order) asymptotic approximation may not be very well located. We shall now assume that a bandwidth of the optimal order $h \asymp n^{-1 / 3}$ has been chosen so as to simplify the discussion of the results. Define the functions

$$
\begin{gathered}
\beta(x)=p^{\prime \prime}(x) \\
s_{p p}(x)=E\left[\Psi_{p p}\left(Z_{i} ; \tau_{0}, p\left(X_{i}\right)\right) \mid X_{i}=x\right]=2\left[\frac{m_{1}(x)}{p(x)^{2}}-\frac{m_{0}(x)}{(1-p(x))^{2}}\right] \\
\mu_{2}(K)=\int u^{2} K(u) d u / 2 \quad ; \quad\|K\|^{2}=\int K(u)^{2} d u .
\end{gathered}
$$

TheOREm 1. Under some regularity conditions, as $n \rightarrow \infty, R=o_{p}\left(n^{-1 / 3}\right)$ in (6) and:

$$
\begin{aligned}
E\left(\mathcal{T}^{*}\right) & \simeq \sqrt{n} h^{2} b_{n 1}+\frac{1}{\sqrt{n} h} b_{2}+o\left(n^{-1 / 3}\right) \\
\operatorname{var}\left(\mathcal{T}^{*}\right) & \simeq v_{0}+o\left(n^{-1 / 3}\right),
\end{aligned}
$$

where $b_{n 1}$ is deterministic and satisfies $b_{n 1} \rightarrow b_{1}$ with

$$
b_{1}=\mu_{2}(K) E\left[s_{p}\left(X_{i}\right) \beta\left(X_{i}\right)\right] \quad ; \quad b_{2}=\|K\|^{2} E\left[s_{p p}\left(X_{i}\right) \frac{p\left(X_{i}\right)\left(1-p\left(X_{i}\right)\right)}{2 f\left(X_{i}\right)}\right] .
$$

The leading smoothing bias term $b_{1}$ can take either sign, since it depends on the covariance between the smoothing bias quantity $\beta(X)$ and on the conditional expectation $s_{p}(X)$. When $p$ is a standard normal c.d.f., $p^{\prime \prime}(x)<0$ for all $x$ and the smoothing bias function is always negative; in this case the direction of the bias is effectively determined by the sign of the treatment effect. The term $b_{2}$ can also take either sign depending on the sign of $s_{p p}(x)$. Suppose there is a constant treatment effect $\tau$ independent of $X$, that $p(x)=1 / 2$ for all $x$, and that $f$ is uniform with range one. Then $b_{2}=\|K\|^{2} \times \tau$, and the sign of $b_{2}$ is determined by the direction of the treatment effect. The correction term in the variance is clearly of smaller order than the squared bias no matter what bandwidth is chosen.

Define the asymptotic mean squared error of the estimator to be [apart from a factor of order $\left.n^{-1}\right]$

$$
A M S E(\widehat{\tau})=E\left(\mathcal{T}^{* 2}\right)=\operatorname{var}\left(\mathcal{T}^{*}\right)+E^{2}\left(\mathcal{T}^{*}\right),
$$


and define an optimal bandwidth $h_{\text {opt }}$ to be a sequence that minimizes $A M S E(\widehat{\tau})$. By Theorem 1,

$$
A M S E(\widehat{\tau})=v_{0}+\left(\sqrt{n} h^{2} b_{1}+\frac{1}{\sqrt{n} h} b_{2}\right)^{2}+o\left(n^{-1 / 3}\right)
$$

and it suffices to minimize the size of the term inside the brackets. If the biases have opposite signs then the optimal bandwidth is going to set

$$
\sqrt{n} h^{2} b_{1}+\frac{1}{\sqrt{n} h} b_{2}=0
$$

and this second order bias will then be of smaller order. Otherwise, the optimal bandwidth will minimize this second order bias and there will be an interior solution to the optimization problem that can be found by calculus. To summarize, we have

$$
h_{o p t}= \begin{cases}\left(\frac{-b_{2}}{b_{1}}\right)^{1 / 3} n^{-1 / 3} & \text { if } \operatorname{sign}\left(b_{2}\right) \neq \operatorname{sign}\left(b_{1}\right) \\ \left(\frac{b_{2}}{2 b_{1}}\right)^{1 / 3} n^{-1 / 3} & \text { if } \operatorname{sign}\left(b_{2}\right)=\operatorname{sign}\left(b_{1}\right) .\end{cases}
$$

A feasible bandwidth selection method can be defined based on estimates of the quantities $b_{j}, j=1,2$, either nonparametric estimates or parametric estimates suggested from some sort of Silverman's rule of thumb idea. ${ }^{4}$

In some semiparametric estimators it has been shown that by using leave-one-out estimators and other devices one can eliminate the degrees of freedom bias terms of order $n^{-1 / 2} h^{-1}$, see for example Hall and Marron (1987) and Linton (1995). Indeed, we have used a leave-one-out estimator here. Unfortunately, it has not completely eliminated the degrees of freedom bias. Instead, we define an explicit bias correction method and show that it does indeed 'knock' this term out and therefore permits a smaller bandwidth and a better AMSE. Specifically, we define the bias-corrected estimator

$$
\widehat{\tau}^{b c}=\widehat{\tau}-\widehat{b}_{n 2},
$$

where

$$
\widehat{b}_{n 2}=\frac{1}{n} \sum_{\substack{i=1 \\ j \neq i}}^{n} \sum_{\substack{j=1 \\ j}}^{n}\left[\frac{Y_{i} \cdot T_{i}}{\widehat{p}\left(X_{i}\right)^{3}}-\frac{Y_{i} \cdot\left(1-T_{i}\right)}{\left[1-\widehat{p}\left(X_{i}\right)\right]^{3}}\right] w_{i j}^{2} \widehat{\varepsilon}_{j}^{2},
$$

where $\widehat{\varepsilon}_{j}=T_{j}-\widehat{p}\left(X_{j}\right)$. Note that the way we have defined the bias correction can be applied to any linear smoother with weights $w_{i j}$. This bias correction is similar conceptually to using $n-1$ instead

\footnotetext{
${ }^{4}$ This would require a model for $m_{j}, p$, and $f$. See Fan and Gijbels $(1996$, p111) for the solution to a similar problem.
} 
of $n$ in estimating a population variance; significantly, in this context we do not need to estimate higher derivatives of the unknown functions, and it follows that the sampling properties of this bias estimator should be relatively good. ${ }^{5}$

The stochastic expansion for $\widehat{\tau}^{b c}$ is the same as that for $\widehat{\tau}$ except for the additional bias correcting term $\widehat{b}$. On computing the moments of the leading terms of this expansion however we find that the bias term $b_{2}$ has been eliminated; we therefore end up with a better trade-off in the mean squared error of this estimator. The largest terms are a squared bias of order $h^{4} n$ and a variance of order $n^{-1} h^{-1}$. This trade-off leads to an optimal bandwidth $h \propto n^{-2 / 5}$ and mean squared error of $n^{-3 / 5}$. Let

$$
\begin{aligned}
\zeta_{i}=\Psi_{p}\left(Z_{i} ; \tau_{0}, p\left(X_{i}\right)\right)-E\left[\Psi_{p}\left(Z_{i} ; \tau_{0}, p\left(X_{i}\right)\right) \mid X_{i}\right] \\
(K * K)(t)=\int K(t) K(t-u) d u \\
\langle f, g\rangle=\int f(t) g(t) d t .
\end{aligned}
$$

Let now $\mathcal{T}=\sqrt{n}\left(\widehat{\tau}^{b c}-\tau_{0}\right)$ and obtain the stochastic expansion $\mathcal{T}=\mathcal{T}^{*}+R$ as in (6).

Theorem 2. Under some regularity conditions, as $n \rightarrow \infty, R=o_{p}\left(n^{-3 / 5}\right)$ in (6) and:

$$
\begin{aligned}
E\left(\mathcal{T}^{*}\right) & \simeq \sqrt{n} h^{2} b_{1}+o\left(n^{-3 / 5}\right) \\
\operatorname{var}\left(\mathcal{T}^{*}\right) & \simeq v_{0}+\frac{1}{n h} v_{1}+o\left(n^{-3 / 5}\right),
\end{aligned}
$$

where

$$
\begin{aligned}
v_{1}= & \|K\|^{2} \times\left\{E\left[\frac{E\left(\varepsilon_{j}^{2} \mid X_{j}\right) E\left(\zeta_{j}^{2} \mid X_{j}\right)}{f\left(X_{j}\right)}\right]+2 E\left[\frac{E^{2}\left(\varepsilon_{j} \zeta_{j} \mid X_{j}\right)}{f\left(X_{j}\right)}\right]\right\} \\
& +\|K * K\|^{2} \times E\left[\frac{3 s_{p p}^{2}\left(X_{j}\right) E^{2}\left(\varepsilon_{j}^{2} \mid X_{j}\right)}{4 f\left(X_{j}\right)}\right] \\
& +\langle K, K * K\rangle \times E\left[\frac{3 s_{p p}\left(X_{j}\right) E\left(\varepsilon_{j}^{2} \mid X_{j}\right) E\left(\varepsilon_{j} \zeta_{j} \mid X_{j}\right)}{f\left(X_{j}\right)}\right] .
\end{aligned}
$$

${ }^{5}$ Effectively, we are estimating the quantity $b_{2} / n h$. We could alternatively estimate $b_{2}$ itself by

$$
\widehat{b}_{2}=\|K\|^{2} \frac{1}{n} \sum_{i=1}^{n} \widehat{s}_{p p}\left(X_{i}\right) \frac{\widehat{p}\left(X_{i}\right)\left(1-\widehat{p}\left(X_{i}\right)\right)}{2 \widehat{f}\left(X_{i}\right)} .
$$

This is just a sample average of nonparametric estimators, and is similar in this respect to a weighted average derivative [in our case second derivatives] estimator. Therefore, under some regularity conditions we can expect it to satisfy $\sqrt{n}\left(\widehat{b}_{2}-b_{2}\right)=O_{p}(1)$. 
This shows that the bias correction can lead to improved mean squared error properties. ${ }^{6}$ In this case,

$$
A M S E\left(\widehat{\tau}^{b c}\right)=v_{0}+n h^{4} b_{1}^{2}+\frac{1}{n h} v_{1}+o\left(n^{-3 / 5}\right)
$$

and the optimal bandwidth is

$$
h_{o p t}=\left(\frac{v_{1}}{4 b_{1}^{2}}\right)^{1 / 5} n^{-2 / 5},
$$

since $b_{1}^{2}, v_{1}$ are both non-negative. This bandwidth is smaller in magnitude than is optimal for the raw estimator $\widehat{\tau}$. A feasible bandwidth selection method can be defined based on estimates of the quantities $b_{1}, v_{1}$, either nonparametric estimates or parametric estimates suggested from some sort of Silverman's rule of thumb idea.

The degrees of freedom bias correction has been analyzed before in other contexts. For example, Jones and Sheather (1991) investigated squared density derivatives, the situation of Hall and Marron (1987). They argued against doing the degrees of freedom bias correction by itself in this case. Their reasoning was that the leading smoothing bias term was always negative, while the degrees of freedom bias term was always positive. Therefore, by a judicious choice of bandwidth one could cancel these terms out. If we applied their method successfully to our problem we would end up with [assuming that $h \asymp n^{-1 / 3}$ ]

$$
A M S E\left(\widehat{\tau}^{J S}\right) \simeq v_{0}+\left(\sqrt{n} h^{4} b_{11}\right)^{2}+\frac{1}{n h} v_{1}
$$

say, where $b_{11}$ is a higher order smoothing bias term [assuming that the underlying functions are smooth enough]. In this case, the correction term is of order $n^{-2 / 3}$, which is even smaller than the order $n^{-3 / 5}$ obtained with our degrees of freedom bias correction. The catch is that in our more complicated model, the signs of the two bias terms are not necessarily opposite and so the Jones and Sheather method is not guaranteed to work and the resulting correction term is then larger than ours. In any case, the Jones and Sheather method requires estimation of higher order derivatives of the regression function and is: (a) unlikely to work well in practice, and (b) against the spirit of our approach.

\footnotetext{
${ }^{6}$ We are happy to report that this finding is partly in agreement with Rothenberg (1984a, p909) who says:
}

"This suggests that correction for bias may be more important than second order efficiency consideration when choosing among estimators."

In our case, correction for bias improves mean squared error. 
We have just presented results concerning the moments of the estimators, but this can also be extended to distributional approximations. In fact, to the relevant order $\widehat{\tau}$ is normally distributed, i.e.,

$$
\operatorname{Pr}\left[\sqrt{n}\left(\widehat{\tau}-\tau_{0}\right) \leq x\right]=\Phi\left(\frac{x-\sqrt{n} h^{2} b_{1}+\frac{1}{\sqrt{n} h} b_{2}}{\sqrt{v_{0}}}\right)+o\left(n^{-1 / 3}\right) .
$$

The approximation for $\sqrt{n}\left(\widehat{\tau}^{b c}-\tau_{0}\right)$ is more complicated because if we require an error rate consistent with our mean squared error [i.e., of order $n^{-3 / 5}$ ] then we will have to include the skewness terms of order $n^{-1 / 2} ;^{7}$ in this case the approximate distribution is not normal in general but can be expressed in terms of the Edgeworth signed measures and the first three cumulant approximations. See Linton (2001) for a computation of this type.

Finally, we remark that the standard errors of $\widehat{\tau}$ also depend on the nonparametric estimator $\widehat{p}(\cdot)$, and there are similar concerns about the small sample properties of these quantities. These standard errors also suffer from a degrees of freedom bias problem, which can be corrected in the same way as we have done for the estimator of $\tau$.

\section{Some Numerical Results}

For comparison we present the optimal rates associated with a variety of semiparametric models that have been studied before. These are all for the univariate case with second order kernels or similar method.

\section{TABLE 1}

Rates of Convergence for Bandwidth and Mean Squared Error Correction

\footnotetext{
${ }^{7}$ In both cases

$$
E\left[\left\{\mathcal{T}^{*}-E\left(\mathcal{T}^{*}\right)\right\}^{3}\right] \simeq O\left(n^{-1 / 2}\right),
$$
}

which is the same magnitude as in parametric models. 


\begin{tabular}{lcc}
\hline \hline Model & Optimal Bandwidth & Optimal MSE Correction \\
\hline \hline 1. Average Derivative & $n^{-2 / 7}$ & $n^{-1 / 7}$ \\
2. Variance Estimation & $n^{-1 / 5}$ & $n^{-3 / 5}$ \\
3. Partially Linear Model & $n^{-2 / 9}$ & $n^{-7 / 9}$ \\
4. Heteroskedastic Linear Regression & $n^{-1 / 5}$ & $n^{-4 / 5}$ \\
5. Variance a Function of Mean & $n^{-2 / 11}$ & $n^{-5 / 11}$ \\
6. Symmetric Location & $n^{-1 / 7}$ & $n^{-4 / 7}$ \\
7. HIR & $n^{-1 / 3}$ & $n^{-1 / 3}$ \\
8. HIR with Bias Correction & $n^{-2 / 5}$ & $n^{-3 / 5}$ \\
\hline \hline
\end{tabular}

Notes. Models 2-6 are given in Linton (1991, Chapter 3). The result for Model 1 is taken from Härdle, Hart, Marron, \& Tsybakov (1992).

The optimal bandwidth for nonparametric regression is of order $n^{-1 / 5}$ and has a consequent MSE of order $n^{-4 / 5}$. Table 1 shows that there is quite a variety of magnitudes for the optimal bandwidth in semiparametric estimation problems; sometimes the optimal bandwidth is bigger but usually it is smaller than the optimal rates for nonparametric estimation. These different rates reflect different magnitudes for bias and variance in these semiparametric functionals.

We investigate the magnitudes of the second order effects in Theorems 1 and 2 and the optimal bandwidth size. We compute the theoretically optimal bandwidths and mean squared errors for the following model.

\section{Design.}

$$
\begin{aligned}
X & \sim U[-0.5,0.5] ; T=1(\beta X+\delta>0) \\
m_{0}(x) & =x ; m_{1}(x)=\tau+m_{0}(x) \\
Y_{0} & =m_{0}(X)+\eta ; Y_{1}=y_{0}+\tau,
\end{aligned}
$$

where $\eta, \delta \sim N(0,1)$ and are mutually independent. We vary the parameters $\tau$ and $\beta$ with $\tau \in$ $\{-2,-1,0,1,2\}$ and $\beta \in\{1,2,3\} .^{8}$

We compute the quantities in Theorem 1 and 2 by simulation methods. Note that $v_{0}$ changes substantially with $\beta$ and less with $\tau$. For example, when $(\beta, \tau)=(1,-2), v_{0}=4.28$, while when $(\beta, \tau)=(1,+2), v_{0}=4.31$. However, when $(\beta, \tau)=(3,-2), v_{0}=29.57$ and when $(\beta, \tau)=(3,+2)$, $v_{0}=42.86$. By contrast, $b_{1}$ and $b_{2}$ are quite small in absolute terms. For $(\beta, \tau)=(1,2),\left(b_{1}, b_{2}\right)=$

${ }^{8}$ The regression $R^{2}$ of $\beta X+\delta$ on $X$ is $R^{2}=2 \beta^{2} /\left(2 \beta^{2}+3\right)$ and of $Y_{j}$ on $X$ is given by the same formula with $\beta=1$, i.e., $R^{2}=0.4$. 
$(0.031,0.484)$, while for $(\beta, \tau)=(3,-2),\left(b_{1}, b_{2}\right)=(12.88,-3.59)$. In most cases $b_{1}$ and $b_{2}$ have opposite signs. The constant $v_{1}$ is very large when $\beta=3$. When $(\beta, \tau)=(1,0), v_{1}=0.5$, while when $(\beta, \tau)=(3,-2), v_{1}=110.36$.

We report the relative root mean squared error against bandwidth $\left[R R M S E=\sqrt{A M S E / v_{0}}\right]$ in the figures below for a sample size of $n=100$ and $n=1000$. The solid line is for the raw estimator and the dashed line is for the bias corrected estimator.

*** Figures 1-2 here ${ }^{* * *}$

The effects of bandwidth on performance are quite clear from these pictures. As discussed earlier there is a bandwidth in this case for which the RRMSE of $\widehat{\tau}$ is exactly equal to one, but this never happens for $\widehat{\tau}^{b c}$. This gives the misleading impression that the un-corrected estimator is better. But of course the error in the expansion for $\widehat{\tau}^{b c}$ is of much smaller order than in $\widehat{\tau}$ - for a meaningful comparison we should include more terms in expansion of $\widehat{\tau}$. It is clear from the pictures that $\widehat{\tau}^{b c}$ has better RRMSE when $h$ is small, but that the estimators have similar RRMSE when $h$ is large.

Note that the Silverman's rule of thumb bandwidth [for Gaussian kernels] $h_{\text {rot }}=1.06 \sigma n^{-1 / 5}$ is for $n=100, h_{\text {rot }}=0.35, n=200, h_{\text {rot }}=0.30, n=500, h_{\text {rot }}=0.25, n=1000, h_{\text {rot }}=0.22$, and $n=10,000, h_{\text {rot }}=0.14$. Another common bandwidth choice is just $0.2 \times$ range, which in this case would result in $h=0.2$. For the small sample size these bandwidths rarely do dreadfully, but such large bandwidths can have disastrous effects in the larger samples.

\section{Conclusions}

Our asymptotic expansions revealed some facts about the HIR estimator. The main thing is that its properties are dominated by bias: one bias term is related to the curvature of the function $p$ and the covariate density $f$, and would naturally be called a smoothing bias; the second bias term is what we have called a degrees of freedom bias. The magnitude of the bias terms can be quite large and their signs are unknown in general. We proposed a simple bias correction that eliminates the degrees of freedom bias term, thereby permitting a smaller bandwidth and consequently better mean squared error. 


\section{Appendix}

Sufficient conditions for consistency and asymptotic normality of semiparametric estimators can be found in numerous places for a variety of nonparametric estimators and estimation criteria. See for example Andrews (1994), Newey \& McFadden (1994), Bickel, Klaassen, Ritov, \& Wellner (1993) etc. Linton (1996b) develops higher order asymptotic expansions for a general class of semiparametric estimators. We will clearly require smoothness conditions on $p, f$. We require that both $p$ and $f$ be bounded away from zero on the compact support of $X$. We also need some moment conditions on $Y_{j i}$. The conditions should imply at least that

$$
\sup _{x \in C}|\widehat{p}(x)-p(x)|=o_{p}\left(n^{-1 / 4}\right)
$$

where $C$ is the support of $X$. Sufficient conditions for this can be found in Masry $(1996 \mathrm{a}, \mathrm{b})$, who actually shows that

$$
\sup _{x \in C}|\widehat{p}(x)-p(x)|=O_{p}\left(h^{2}\right)+O_{p}\left(\sqrt{\frac{\log n}{n h}}\right),
$$

which is $O_{p}\left(n^{-1 / 3} \sqrt{\log n}\right)$ when $h \asymp n^{-1 / 3}$ and $O_{p}\left(n^{-3 / 10} \sqrt{\log n}\right)$ when $h \asymp n^{-2 / 5}$; in either case this magnitude is $o_{p}\left(n^{-1 / 4}\right)$ as required. We also use the decomposition

$$
\widehat{p}\left(X_{i}\right)-p\left(X_{i}\right)=\sum_{j \neq i} w_{i j} \varepsilon_{j}+\beta_{n}\left(X_{i}\right)
$$

where $w_{i j}$ are the smoothing weights that just depend on the covariates $X_{1}, \ldots, X_{n}$, while $\beta_{n}\left(X_{i}\right)=$ $E\left[\widehat{p}\left(X_{i}\right) \mid X_{1}, \ldots, X_{n}\right]-p\left(X_{i}\right)$ is the conditional smoothing bias that also just depends on the covariates $X_{1}, \ldots, X_{n}$. It can be shown that $h^{-2} \beta_{n}(x) \rightarrow^{p} \beta(x)$ and that this convergence is uniform, see for example Masry (1996a,b). In fact,

$$
\begin{aligned}
& E\left[\left(\widehat{p}\left(X_{i}\right)-p\left(X_{i}\right)\right)^{2} \mid X_{1}, \ldots, X_{n}\right] \\
\simeq & \frac{1}{n h}\|K\|^{2} \frac{p\left(X_{i}\right)\left(1-p\left(X_{i}\right)\right)}{f\left(X_{i}\right)}+\frac{h^{4}}{4} \mu_{2}^{2}(K) \beta^{2}\left(X_{i}\right) \equiv M_{n}\left(X_{i}\right) .
\end{aligned}
$$

See the book of Fan and Gijbels (1996) for more discussion.

Proof of Theorem 1. By a geometric series expansion

$$
\sqrt{n}\left(\widehat{\tau}-\tau_{0}\right)=\frac{1}{\sqrt{n}} \sum_{i=1}^{n} \Psi\left(Z_{i} ; \tau_{0}, p\left(X_{i}\right)\right)+\frac{1}{\sqrt{n}} \sum_{i=1}^{n} \Psi_{p}\left(Z_{i} ; \tau_{0}, p\left(X_{i}\right)\right)\left(\widehat{p}\left(X_{i}\right)-p\left(X_{i}\right)\right)
$$




$$
\begin{aligned}
& +\frac{1}{2 \sqrt{n}} \sum_{i=1}^{n} \Psi_{p p}\left(Z_{i} ; \tau_{0}, p\left(X_{i}\right)\right)\left(\widehat{p}\left(X_{i}\right)-p\left(X_{i}\right)\right)^{2} \\
& +\frac{1}{6 \sqrt{n}} \sum_{i=1}^{n} \Psi_{p p p}\left(Z_{i} ; \tau_{0}, p\left(X_{i}\right)\right)\left(\widehat{p}\left(X_{i}\right)-p\left(X_{i}\right)\right)^{3} \\
& +\frac{1}{24 \sqrt{n}} \sum_{i=1}^{n} \Psi_{p p p p}\left(Z_{i} ; \tau_{0}, p\left(X_{i}\right)\right)\left(\widehat{p}\left(X_{i}\right)-p\left(X_{i}\right)\right)^{4}+o_{p}\left(n^{-3 / 4}\right) .
\end{aligned}
$$

The magnitude of the remainder in (13) follows from (10) and because the derivatives of $\Psi$ with respect to $p$ are dominated by a function with finite moment [since $p$ is bounded away from zero].

When $h \asymp n^{-1 / 3}$ and we only require an expansion out to order $n^{-1 / 3}$, we can further drop the cubic and quartic terms to obtain

$$
\begin{aligned}
\sqrt{n}\left(\widehat{\tau}-\tau_{0}\right)= & \frac{1}{\sqrt{n}} \sum_{i=1}^{n} \Psi\left(Z_{i} ; \tau_{0}, p\left(X_{i}\right)\right)+\frac{1}{\sqrt{n}} \sum_{i=1}^{n} s_{p}\left(X_{i}\right)\left(\widehat{p}\left(X_{i}\right)-p\left(X_{i}\right)\right) \\
& +\frac{1}{\sqrt{n}} \sum_{i=1}^{n} \zeta_{i} \cdot\left(\widehat{p}\left(X_{i}\right)-p\left(X_{i}\right)\right)+\frac{1}{2 \sqrt{n}} \sum_{i=1}^{n} s_{p p}\left(X_{i}\right)\left(\widehat{p}\left(X_{i}\right)-p\left(X_{i}\right)\right)^{2} \\
& +\frac{1}{2 \sqrt{n}} \sum_{i=1}^{n} \xi_{i} \cdot\left(\widehat{p}\left(X_{i}\right)-p\left(X_{i}\right)\right)^{2}+o_{p}\left(n^{-1 / 3}\right) \\
\equiv & J_{1}+J_{2}+J_{3}+J_{4}+o_{p}\left(n^{-1 / 3}\right)
\end{aligned}
$$

where the random variables $\zeta_{i}=\Psi_{p}\left(Z_{i} ; \tau_{0}, p\left(X_{i}\right)\right)-E\left[\Psi_{p}\left(Z_{i} ; \tau_{0}, p\left(X_{i}\right)\right) \mid X_{i}\right]$ and $\xi_{i}=\Psi_{p p}\left(Z_{i} ; \tau_{0}, p\left(X_{i}\right)\right)-E\left[\Psi_{p p}\left(Z_{i} ; \tau_{0}, p\left(X_{i}\right)\right) \mid X_{i}\right]$ are both i.i.d. and conditional mean zero given $X_{j}$.

We then write

$$
\begin{aligned}
J_{1} & =\frac{1}{\sqrt{n}} \sum_{i=1}^{n} s_{p}\left(X_{i}\right)\left(\widehat{p}\left(X_{i}\right)-p\left(X_{i}\right)\right) \\
& =\frac{1}{\sqrt{n}} \sum_{j=1}^{n} s_{p}\left(X_{j}\right) \varepsilon_{j}+\frac{1}{\sqrt{n}} \sum_{j=1}^{n} \varepsilon_{j}\left[\sum_{i \neq j} w_{i j} s_{p}\left(X_{i}\right)-s_{p}\left(X_{j}\right)\right]+\frac{1}{\sqrt{n}} \sum_{i=1}^{n} s_{p}\left(X_{i}\right) \beta_{n}\left(X_{i}\right) \\
& \equiv J_{11}+J_{12}+J_{13},
\end{aligned}
$$

where $J_{11}=O_{p}(1)$ and asymptotically normal [also jointly asymptotically normal with the leading term in our expansion, $\left.n^{-1 / 2} \sum_{i=1}^{n} \Psi\left(Z_{i} ; \tau_{0}, p\left(X_{i}\right)\right)\right]$, the term $J_{12}$ is mean zero and has variance of the same magnitude as $E\left[\sum_{i \neq j} w_{i j} s_{p}\left(X_{i}\right)-s_{p}\left(X_{j}\right)\right]^{2}$, this we expect to be $O\left(h^{4}\right)$. The reason is that $w_{i j}$ are approximately symmetric [see Linton (2001b)] and so $\sum_{i \neq j} w_{i j} s_{p}\left(X_{i}\right)-s_{p}\left(X_{j}\right)$ is rather like $\sum_{i \neq j} w_{j i} s_{p}\left(X_{i}\right)-s_{p}\left(X_{j}\right)$ in terms of its magnitude, and this latter quantity is just the bias function from smoothing $s_{p}\left(X_{i}\right)$ against $X_{i}$. Therefore, $J_{12}=O_{p}\left(h^{2}\right)$. The term $J_{13}$ is a bias term with magnitude $h^{2} \sqrt{n}$ and variance also $h^{4}$. This term contributes to the second approximation of 
Theorem 1, specifically

$$
h^{-2} \frac{1}{n} \sum_{i=1}^{n} s_{p}\left(X_{i}\right) \beta_{n}\left(X_{i}\right) \rightarrow^{p} b_{1}
$$

as stated.

We next turn to the term

$$
\begin{aligned}
J_{2} & =\frac{1}{\sqrt{n}} \sum_{i=1}^{n} \zeta_{i} \cdot\left(\widehat{p}\left(X_{i}\right)-p\left(X_{i}\right)\right) \\
& =\frac{1}{\sqrt{n}} \sum_{i=1}^{n} \zeta_{i} \sum_{j \neq i} w_{i j} \varepsilon_{j}+\frac{1}{\sqrt{n}} \sum_{i=1}^{n} \zeta_{i} \beta_{n}\left(X_{i}\right) \equiv J_{21}+J_{22},
\end{aligned}
$$

where $J_{21}$ is a second order degenerate U-statistic that has mean zero and variance of order $n^{-1} h^{-1}$; it is also uncorrelated with the leading terms. The second term, $J_{22}$, is mean zero and $O_{p}\left(h^{2}\right)$ and hence of smaller order.

We next turn our attention to the term $J_{3}$. First,

$$
\begin{aligned}
J_{3} & =\frac{1}{\sqrt{n}} \sum_{i=1}^{n} s_{p p}\left(X_{i}\right)\left(\widehat{p}\left(X_{i}\right)-p\left(X_{i}\right)\right)^{2} \\
& =\frac{1}{\sqrt{n}} \sum_{i=1}^{n} s_{p p}\left(X_{i}\right) E\left[\left(\widehat{p}\left(X_{i}\right)-p\left(X_{i}\right)\right)^{2} \mid X_{1}, \ldots, X_{n}\right]+O_{p}\left(n^{-1 / 2} h^{-1 / 2}\right) \\
& =\frac{1}{h \sqrt{n}}\|K\|^{2} \frac{1}{n} \sum_{i=1}^{n} s_{p p}\left(X_{i}\right) \frac{p\left(X_{i}\right)\left(1-p\left(X_{i}\right)\right)}{f\left(X_{i}\right)}+O_{p}\left(h^{4} \sqrt{n}\right)+O_{p}\left(n^{-1 / 2} h^{-1 / 2}\right),
\end{aligned}
$$

where we have substituted in the expression for $E\left[\left(\widehat{p}\left(X_{i}\right)-p\left(X_{i}\right)\right)^{2} \mid X_{1}, \ldots, X_{n}\right]$. Finally, taking probability limits

$$
h \sqrt{n} J_{3}=b_{2}+o_{p}(1)
$$

using the fact that $h^{5} n \rightarrow 0$. The omitted term in (16) is mean zero and uncorrelated with the lead, so only its variance, which is of order $n^{-1} h^{-1}$ contributes to the mean squared error; with the bandwidth magnitude of Theorem 1, it is of smaller order. To make clearer the claim in (16), and in anticipation of the proof of Theorem 2 where this term is important, we give another argument here. Using (12), we can write

$$
J_{3}=\frac{1}{\sqrt{n}} \sum_{i=1}^{n} s_{p p}\left(X_{i}\right)\left[\sum_{j \neq i} w_{i j} \varepsilon_{j}+O_{p}\left(h^{2}\right)\right]^{2}=\frac{1}{\sqrt{n}} \sum_{i=1}^{n} s_{p p}\left(X_{i}\right)\left[\sum_{j \neq i} w_{i j} \varepsilon_{j}\right]^{2}+O_{p}\left(h^{4} \sqrt{n}\right)
$$




$$
\begin{aligned}
\simeq & \frac{1}{\sqrt{n}} \sum_{i=1}^{n} s_{p p}\left(X_{i}\right) \sum_{j \neq i} w_{i j}^{2} E\left(\varepsilon_{j}^{2} \mid X_{j}\right)+\frac{1}{\sqrt{n}} \sum_{i=1}^{n} s_{p p}\left(X_{i}\right) \sum_{j \neq i} w_{i j}^{2}\left[\varepsilon_{j}^{2}-E\left(\varepsilon_{j}^{2} \mid X_{j}\right)\right] \\
& +\frac{1}{\sqrt{n}} \sum_{i=1}^{n} s_{p p}\left(X_{i}\right) \sum_{\substack{j \neq i \\
j \neq l}} \sum_{\substack{l \neq i \\
j \neq l}} w_{i j} w_{i l} \varepsilon_{j} \varepsilon_{l} \equiv J_{31}+J_{32}+J_{33} .
\end{aligned}
$$

The first term $J_{31}$ is the leading bias term of order $h^{-1} n^{-1 / 2}$ analyzed above. The second term $J_{32}$ is mean zero and is of order $n^{-1} h^{-1}$ in probability so is insignificant in both Theorem 1 and 2 . The third term, $J_{33}$, is mean zero and $O_{p}\left(n^{-1 / 2} h^{-1 / 2}\right)$. We can rewrite this term

$$
\begin{aligned}
\frac{1}{\sqrt{n}} \sum_{i=1}^{n} s_{p p}\left(X_{i}\right) \sum_{\substack{j \neq i \\
j \neq l}} \sum_{\substack{l \neq i \\
j}} w_{i j} w_{i l} \varepsilon_{j} \varepsilon_{l} & =\sum_{j \neq l} \sum_{j}\left(\frac{1}{\sqrt{n}} \sum_{i=1}^{n} s_{p p}\left(X_{i}\right) w_{i j} w_{i l}\right) \varepsilon_{j} \varepsilon_{l} \\
& \simeq \frac{1}{n \sqrt{n} h} \sum_{j \neq l}(K * K)\left(\frac{X_{j}-X_{l}}{h}\right) \frac{s_{p p}\left(X_{j}\right)}{f\left(X_{j}\right)} \varepsilon_{j} \varepsilon_{l},
\end{aligned}
$$

where $K * K(u)=\int K(t-u) K(t) d t$. See Linton (1995) for a similar calculation. This term does not feature in the expansion of Theorem 1 [but does contribute in Theorem 2, see below].

Finally, it is easy to see that

$$
J_{4}=\frac{1}{2 \sqrt{n}} \sum_{i=1}^{n} \xi_{i} \cdot\left(\widehat{p}\left(X_{i}\right)-p\left(X_{i}\right)\right)^{2}=O_{p}\left(h^{4}+n^{-1} h^{-1}\right) .
$$

Specifically, because we are using a leave-one-out estimator, $\xi_{i}$ is independent of $\widehat{p}\left(X_{i}\right)-p\left(X_{i}\right)$ conditional on $X_{i}$. Therefore, this term is mean zero and its order in probability is the same as $E M_{n}\left(X_{i}\right)$.

In conclusion we have

$$
\begin{aligned}
\sqrt{n}\left(\widehat{\tau}-\tau_{0}\right) \simeq & \frac{1}{\sqrt{n}} \sum_{i=1}^{n} \Psi\left(Z_{i} ; \tau_{0}, p\left(X_{i}\right)\right)+s_{p}\left(X_{i}\right) \varepsilon_{i} \quad\left[=O_{p}(1)\right] \\
& +\frac{1}{\sqrt{n}} \sum_{j=1}^{n} \varepsilon_{j}\left[\sum_{i \neq j} w_{i j} s_{p}\left(X_{i}\right)-s_{p}\left(X_{j}\right)\right] \quad\left[=O_{p}\left(h^{2}\right)\right] \\
& +\sum_{i \neq j} \sum_{n} \varphi_{i}\left(Z_{i}, Z_{j}\right) \quad\left[=O_{p}\left(n^{-1 / 2} h^{-1 / 2}\right)\right] \\
& +\frac{1}{\sqrt{n}} \sum_{i=1}^{n} s_{p}\left(X_{i}\right) \beta_{n}\left(X_{i}\right) \quad\left[=O_{p}\left(h^{2} \sqrt{n}\right)\right] \\
& +\frac{1}{2 \sqrt{n}} \sum_{i=1}^{n} s_{p p}\left(X_{i}\right) M_{n}\left(X_{i}\right) \quad\left[=O_{p}\left(h^{4} \sqrt{n}\right)+O_{p}\left(n^{-1 / 2} h^{-1}\right)\right]
\end{aligned}
$$


where

$$
\varphi_{n}\left(Z_{i}, Z_{j}\right)=\frac{1}{n h \sqrt{n}} \frac{1}{f\left(X_{i}\right)}\left[K\left(\frac{X_{i}-X_{j}}{h}\right) \zeta_{i} \varepsilon_{j}+\frac{1}{2}(K * K)\left(\frac{X_{i}-X_{j}}{h}\right) s_{p p}\left(X_{i}\right) \varepsilon_{i} \varepsilon_{j}\right] .
$$

Clearly, $E\left[\varphi_{n}\left(Z_{i}, Z_{j}\right) \mid Z_{i}\right]=E\left[\varphi_{n}\left(Z_{i}, Z_{j}\right) \mid Z_{j}\right]=0$ and $\sum_{i \neq j} \varphi_{n}\left(Z_{i}, Z_{j}\right)$ is a degenerate weighted Ustatistic of the stated order in probability. Because this term is uncorrelated with the leading term it does not contribute to the mean squared error expansion of Theorem 1. Likewise the $O_{p}\left(h^{2}\right)$ term does not contribute because it is mean zero. Therefore, the leading terms in the mean squared error expansion come from

$$
\begin{aligned}
\sqrt{n}\left(\widehat{\tau}-\tau_{0}\right) \simeq & \frac{1}{\sqrt{n}} \sum_{i=1}^{n}\left[\Psi\left(Z_{i} ; \tau_{0}, p\left(X_{i}\right)\right)+s_{p}\left(X_{i}\right) \varepsilon_{i}\right] \\
& +\frac{1}{\sqrt{n}} \sum_{i=1}^{n} s_{p}\left(X_{i}\right) \beta_{n}\left(X_{i}\right)+\frac{1}{2 \sqrt{n}} \sum_{i=1}^{n} s_{p p}\left(X_{i}\right) M_{n}\left(X_{i}\right) .
\end{aligned}
$$

Proof of Theorem 2. In this case we have $h \propto n^{-2 / 5}$ and require a mean squared error expansion upto order $n^{-3 / 5}$.

Write $\widehat{b}_{2}=h \sqrt{n} \widehat{b}_{n 2}$, where recall the target quantity

$$
b_{2}=\|K\|^{2} E\left[s_{p p}\left(X_{i}\right) \frac{p\left(X_{i}\right)\left(1-p\left(X_{i}\right)\right)}{2 f\left(X_{i}\right)}\right] .
$$

First of all we suppose that

$$
\sqrt{n}\left(\widehat{b}_{2}-b_{2}\right)=\frac{1}{\sqrt{n}} \sum_{i=1}^{n} \psi\left(X_{i}\right)+o_{p}(1)
$$

where $E \psi\left(X_{i}\right)=0$ and $\operatorname{var}\left(\psi\left(X_{i}\right)\right)<\infty$. This can be justified by a lengthy argument using standard techniques of semiparametric estimation, see for example Andrews (1994), Newey and McFadden (1994) etc. The consequence of (18) is that

$$
\begin{aligned}
\sqrt{n}\left(\widehat{\tau}^{b c}-\tau_{0}\right) & =\sqrt{n}\left(\widehat{\tau}-\tau_{0}\right)-\frac{b_{2}}{h \sqrt{n}}\left(1+O_{p}\left(n^{-1 / 2}\right)\right) \\
& =\sqrt{n}\left(\widehat{\tau}-\tau_{0}\right)-\frac{b_{2}}{h \sqrt{n}}+O_{p}\left(n^{-3 / 5}\right),
\end{aligned}
$$

where the $O_{p}\left(n^{-3 / 5}\right)$ term is mean zero. Furthermore, this remainder term is uncorrelated with the leading term because $E\left[\Psi\left(Z_{i} ; \tau_{0}, p\left(X_{i}\right)\right)+s_{p}\left(X_{i}\right) \varepsilon_{i} \mid X_{i}\right]=0$, and therefore this term does not contribute to the mean squared error expansion. 
We must examine (13) again because the bandwidth magnitude is different from Theorem 1 . The term

$$
\frac{1}{6 \sqrt{n}} \sum_{i=1}^{n} \Psi_{p p p}\left(Z_{i} ; \tau_{0}, p\left(X_{i}\right)\right)\left(\widehat{p}\left(X_{i}\right)-p\left(X_{i}\right)\right)^{3}=O_{p}\left(n^{-2 / 5}(\log n)^{3 / 2}\right),
$$

and in principle must be analyzed in this case. It can be shown that it does not contribute to the mean squared error because essentially the correlations with leading terms are of smaller order [the square of this term is obviously $\left.n^{-3 / 5}\right]$.

In conclusion,

$$
\begin{aligned}
\sqrt{n}\left(\widehat{\tau}^{b c}-\tau_{0}\right) \simeq & \frac{1}{\sqrt{n}} \sum_{i=1}^{n} \Psi\left(Z_{i} ; \tau_{0}, p\left(X_{i}\right)\right)+s_{p}\left(X_{i}\right) \varepsilon_{i} \\
& +\sum_{i \neq j} \sum_{n}\left(Z_{i}, Z_{j}\right)+\frac{1}{\sqrt{n}} \sum_{i=1}^{n} s_{p}\left(X_{i}\right) \beta_{n}\left(X_{i}\right)
\end{aligned}
$$

where omitted terms do not contribute to the mean squared error to order $n^{-3 / 5}$. Finally,

$$
\operatorname{var}\left[\sum_{i \neq j} \sum_{n}\left(Z_{i}, Z_{j}\right)\right]=n(n-1) E\left[\varphi_{n}^{2}\left(Z_{i}, Z_{j}\right)\right]+2 n(n-1) E\left[\varphi_{n}\left(Z_{i}, Z_{j}\right) \varphi_{n}\left(Z_{j}, Z_{i}\right)\right]
$$

where by the law of iterated expectations

$$
\begin{aligned}
E\left[\varphi_{n}^{2}\left(Z_{i}, Z_{j}\right)\right]= & \frac{1}{n^{3} h^{2}} E\left[\frac{1}{f^{2}\left(X_{i}\right)} K^{2}\left(\frac{X_{i}-X_{j}}{h}\right) E\left[\zeta_{i}^{2} \mid X_{i}\right] E\left[\varepsilon_{j}^{2} \mid X_{j}\right]\right] \\
& +\frac{1}{4 n^{3} h^{2}} E\left[\frac{1}{f^{2}\left(X_{i}\right)}(K * K)^{2}\left(\frac{X_{i}-X_{j}}{h}\right) s_{p p}^{2}\left(X_{i}\right) E\left[\varepsilon_{i}^{2} \mid X_{i}\right] E\left[\varepsilon_{j}^{2} \mid X_{j}\right]\right] \\
+ & \frac{1}{n^{3} h^{2}} E\left[\frac{1}{f^{2}\left(X_{i}\right)}(K * K) \times K\left(\frac{X_{i}-X_{j}}{h}\right) s_{p p}\left(X_{i}\right) E\left[\zeta_{i} \varepsilon_{i} \mid X_{i}\right] E\left[\varepsilon_{j}^{2} \mid X_{j}\right]\right] \\
E\left[\varphi_{n}\left(Z_{i}, Z_{j}\right) \varphi_{n}\left(Z_{j}, Z_{i}\right)\right]= & \frac{1}{n^{3} h^{2}} E\left[\frac{1}{f\left(X_{i}\right) f\left(X_{j}\right)} K^{2}\left(\frac{X_{i}-X_{j}}{h}\right) E\left[\zeta_{i} \varepsilon_{i} \mid X_{i}\right] E\left[\zeta_{j} \varepsilon_{j} \mid X_{j}\right]\right] \\
& +\frac{1}{4 n^{3} h^{2}} E\left[\frac{s_{p p}\left(X_{i}\right) s_{p p}\left(X_{j}\right)}{f\left(X_{i}\right) f\left(X_{j}\right)}(K * K)^{2}\left(\frac{X_{i}-X_{j}}{h}\right) E\left[\varepsilon_{i}^{2} \mid X_{i}\right] E\left[\varepsilon_{j}^{2} \mid X_{j}\right]\right] \\
& \frac{1}{n^{3} h^{2}} E\left[\frac{s_{p p}\left(X_{i}\right)}{f\left(X_{i}\right) f\left(X_{j}\right)}(K \times(K * K))\left(\frac{X_{i}-X_{j}}{h}\right) E\left[\zeta_{i} \varepsilon_{i} \mid X_{i}\right] E\left[\varepsilon_{j}^{2} \mid X_{j}\right]\right] .
\end{aligned}
$$

We use the fact that $K * K$ is symmetric. Then take expectations with respect to $X_{j}$ and use the localizing operation of $K$ and $K * K$ to see that

$$
n(n-1) E\left[\varphi_{n}^{2}\left(Z_{i}, Z_{j}\right)\right] \simeq \frac{1}{n h}\|K\|^{2} E\left[\frac{E\left[\zeta_{i}^{2} \mid X_{i}\right] E\left[\varepsilon_{i}^{2} \mid X_{i}\right]}{f\left(X_{i}\right)}\right]
$$




$$
\begin{aligned}
+ & \frac{1}{4 n h}\|K * K\|^{2} E\left[\frac{s_{p p}^{2}\left(X_{i}\right) E^{2}\left[\varepsilon_{i}^{2} \mid X_{i}\right]}{f\left(X_{i}\right)}\right] \\
+ & \frac{1}{n h}\langle K, K * K\rangle E\left[\frac{s_{p p}\left(X_{i}\right) E\left[\zeta_{i} \varepsilon_{i} \mid X_{i}\right] E\left[\varepsilon_{i}^{2} \mid X_{i}\right]}{f\left(X_{i}\right)}\right] \\
2 n(n-1) E\left[\varphi_{n}\left(Z_{i}, Z_{j}\right) \varphi_{n}\left(Z_{j}, Z_{i}\right)\right]= & \frac{2}{n h}\|K\|^{2} E\left[\frac{E^{2}\left[\zeta_{i} \varepsilon_{i} \mid X_{i}\right]}{f\left(X_{i}\right)}\right] \\
& +\frac{1}{2 n h}\|K * K\|^{2} E\left[\frac{s_{p p}^{2}\left(X_{i}\right) E^{2}\left[\varepsilon_{i}^{2} \mid X_{i}\right]}{f\left(X_{i}\right)}\right] \\
& \frac{2}{n h}\langle K, K * K\rangle E\left[\frac{s_{p p}\left(X_{i}\right) E\left[\zeta_{i} \varepsilon_{i} \mid X_{i}\right] E\left[\varepsilon_{i}^{2} \mid X_{i}\right]}{f\left(X_{i}\right)}\right] .
\end{aligned}
$$

Therefore, the result is as stated.

\section{REFERENCES}

Andrews, D.W.K., (1994). Asymptotics for semiparametric econometric models via stochastic equicontinuity. Econometrica 62, 43-72.

Bickel, P. J., Klaassen, C. A. J., Ritov, Y. and J. A. Wellner (1993). Efficient and adaptive estimation for semiparametric models. The John Hopkins University Press, Baltimore and London.

Carroll, R.J., and W. Härdle, (1989). Second Order Effects in Semiparametric Weighted Least Squares Regression. Statistics, 2, 179-186.

Cochran, W.G. (1968). The effectiveness of adjustment by subclassification in removing bias in observational studies. Biometrics, 24, 295-313.

Fan, J., and I. Gijbels (1996): Local Polynomial Modelling and Its Applications Chapman and Hall.

Hall, P., and J.S. Marron (1987). Estimation of Integrated Squared Density Derivatives. Statistics and Probability Letters 6, 109-115.

Härdle, W., J. Hart, J. S. Marron, and A. B. Tsybakov (1992). Bandwidth Choice for Average Derivative Estimation. Journal of the American Statistical Association, 87, 218-226.

Härdle, W., and A. B. Tsybakov (1993). How sensitive are Average Derivatives. Journal of Econometrics, 58, 31-48. 
Heckman, J., H. Ichimura, J. Smith and P. Todd (1998). Characterization of Selection Bias Using Experimental Data. Econometrica, 66, 1017-1098.

Heckman, J., H. Ichimura, and P. Todd (1998). Matching as an Econometric Estimator. Review of Economic Studies, 65, 261-294.

Hirano, K., G. Imbens, G. Ridder, (2000). Efficient Estimation of Average Treatment Effects using the Estimated Propensity Score. NBER Technical Working Paper 251.

Hsieh, D.A., and C.F. Manski (1987). Monte Carlo Evidence on Adaptive Maximum Likelihood Estimation of a Regression. Annals of Statistics, 15, 541-551.

Jones, M.C., and S.J. Sheather (1991). Using non-Stochastic terms to Advantage in Kernel-based Estimation of Integrated Squared Density Derivatives. Statistics and Probability Letters 11, $511-514$.

Linton, O.B. (1991). Edgeworth Approximation in Semiparametric Regression Models. PhD Thesis, Department of Economics, University of California at Berkeley.

Linton, O.B. (1995). Second Order Approximation in the Partially Linear Regression Model. Econometrica $63,1079-1112$.

Linton, O.B. (1996a). Second order approximation in a linear regression with heteroskedasticity of unknown form. Econometric Reviews 15, 1-32.

Linton, O.B. (1996b). Edgeworth Approximation for MINPIN Estimators in Semiparametric Regression Models. Econometric Theory 12, 30-60.

Linton, O.B. (2001a). Edgeworth approximations for semiparametric instrumental variable estimator and test statistics. Journal of Econometrics 106, 325-368.

Linton, O.B. (2001b). Symmetrizing and unitizing transformations for linear smoothing weights. Computational Statistics 16, 153-164.

Masry, E. (1996a). Multivariate local polynomial regression for time series: Uniform strong consistency and rates. Journal of Time Series Analysis 17, 571-599.

Masry, E. (1996b). Multivariate regression estimation Local polynomial fitting for time series. Stochastic Processes and their Applications 65, 81-101. 
Nagar, A.L. (1959). The bias and moment matrix of the general $k$-class estimator of the parameters in simultaneous equations. Econometrica 27, 575-595.

Newey, W.K. and D.F. McFadden (1994). Large sample estimation and hypothesis testing. in Handbook of Econometrics, vol. IV, eds. D.F. McFadden and R.F. Engle III. North Holland.

Nishiyama, Y., and Robinson, P. M. (2000). Edgeworth expansions for semiparametric averaged derivatives. Econometrica 68, 931-980.

Powell, J.L., and T.M. Stoker (1996). Optimal bandwidth choice for density-weighted averages. Journal of Econometrics 75, 291-316.

Robinson, P. M. (1995). The normal approximation for semiparametric averaged derivatives. Econometrica $63,667-680$.

Rosenbaum, P. and Rubin, D.B. (1983). The central role of the propensity score in observational studies for causal effects. Biometrika, 70, pp. 41-55.

Rothenberg, T., (1984a). Approximating the Distributions of Econometric Estimators and Test Statistics. Ch.14 in: Handbook of Econometrics, vol 2, ed. Z. Griliches and M.Intriligator. North Holland.

Rothenberg, T., (1984b). Approximate Normality of Generalized Least Squares Estimates. Econometrica, 52, 811-825.

Rothenberg, T., (1984c). Hypothesis Testing in Linear Models when the Error Covariance Matrix is Nonscalar. Econometrica, 52, 827-842.

Rothenberg, T., (1988). Approximate Power Functions for some Robust Tests of Regression Coefficients. Econometrica, 56, 997-1019.

Srinavasan, T.N. (1970). Approximations to Finite Sample Moments of Estimators whose Exact Sampling Distributions are unknown. Econometrica, 38, 533-541.

Xiao, Z. and O.B. Linton (2001). Second order approximation for an adaptive estimator in a linear regression. Econometric Theory 17, 984-1024.

Xiao, Z. and P.C.B. Phillips (1996). Higher order approximation for a frequency domain regression estimator. Journal of Econometrics, 86, 297-336. 


\section{Figures}

The figures show the relative root mean squared error against bandwidth $\left[R R M S E=\sqrt{A M S E / v_{0}}\right]$. The solid line is for the raw estimator and the dashed line is for the bias corrected estimator.The figures show RRMSE against bandwidth $h$. Figure 1 is for $n=100$ and Figure 2 is for $n=1000$ 


$$
\tau=-2 \beta=1
$$

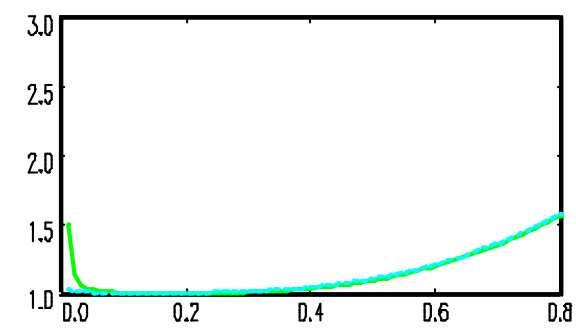

$\tau=-1 \quad \beta=1$
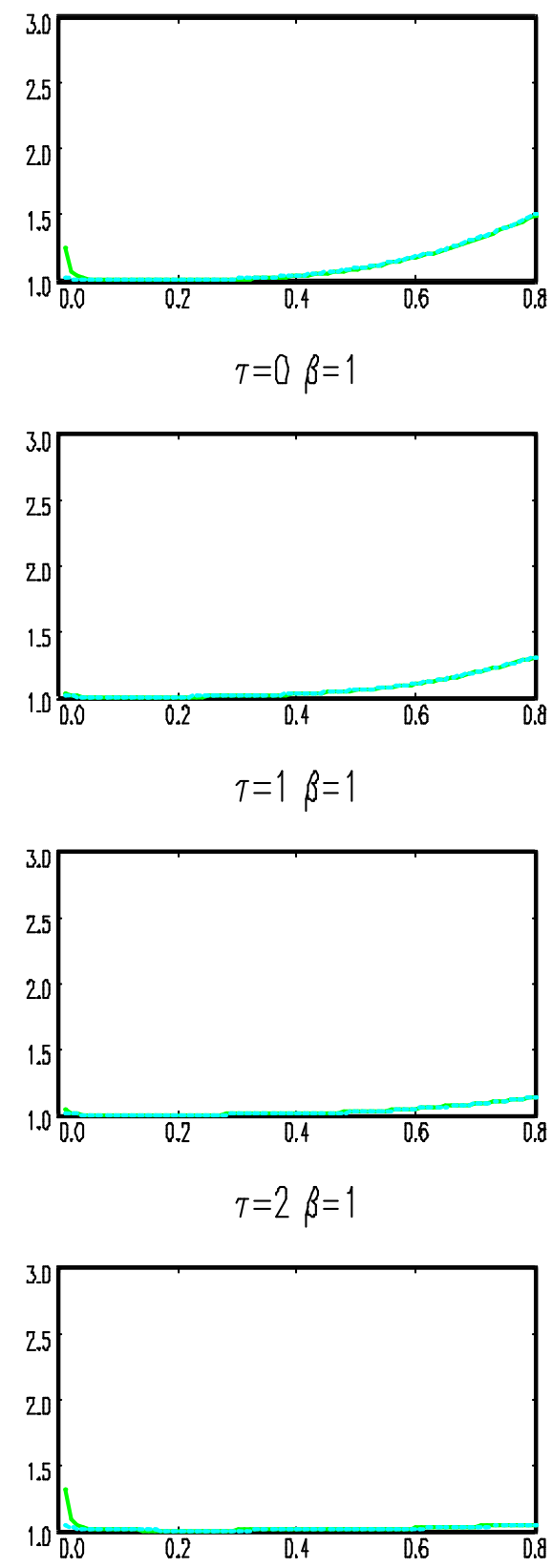

$\tau=-2 \beta=2$

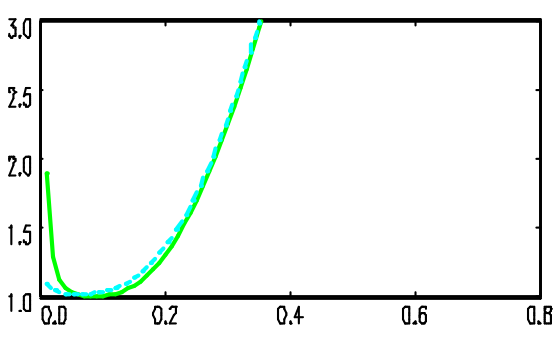

$\tau=-1 \quad \beta=2$

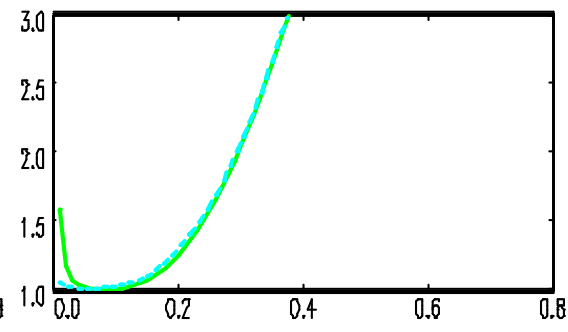

$$
\tau=0 \quad \beta=2
$$

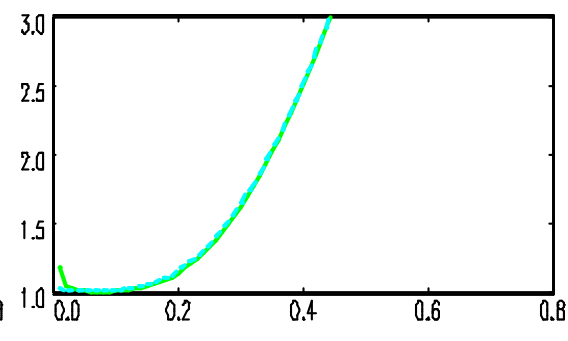

$\tau=1 \beta=2$

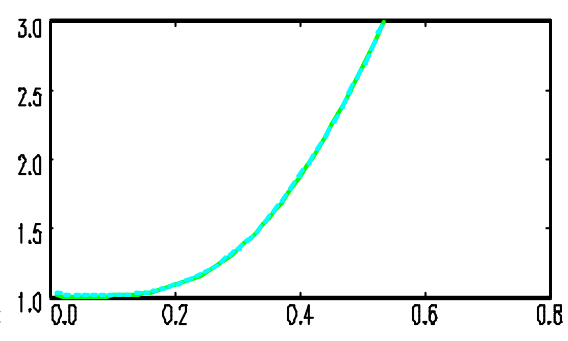

$\tau=2 \beta=2$

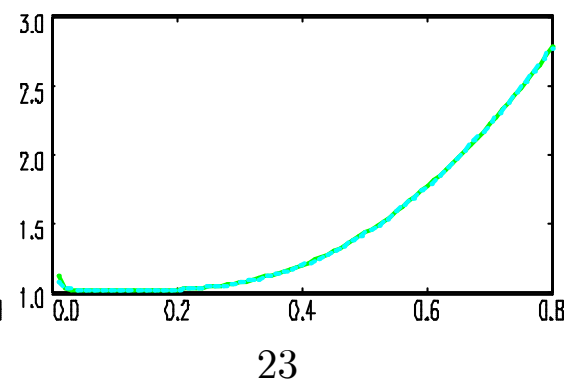

$T=-2 \beta=3$

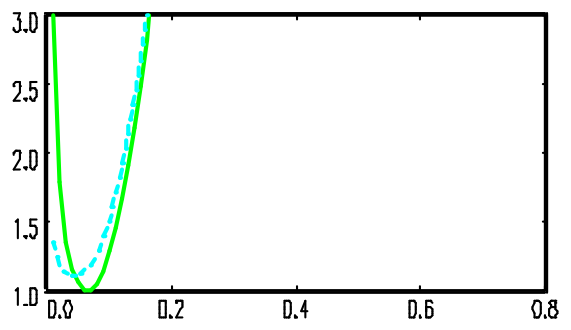

$\tau=-1 \quad \beta=3$

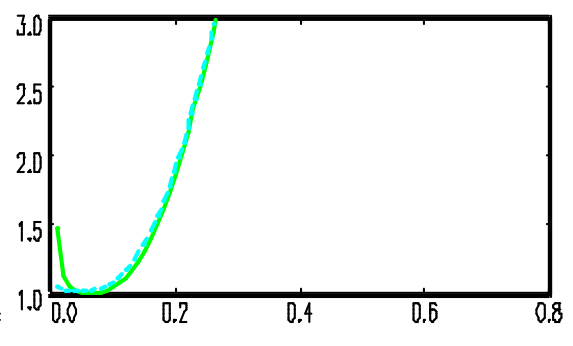

$\tau=0 \beta=3$

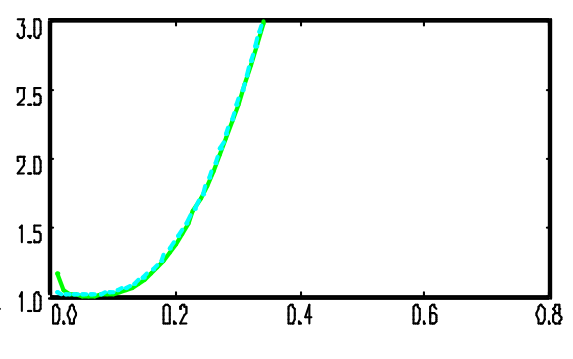

$\tau=1 \quad \beta=3$

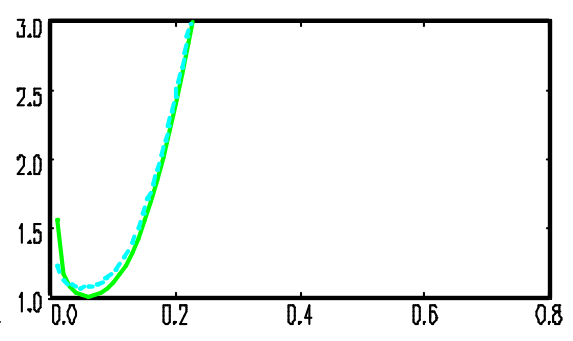

$\tau=2 \beta=3$

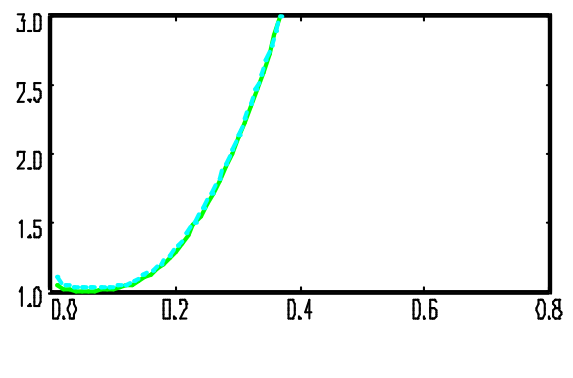


$\tau=-2 \beta=1$

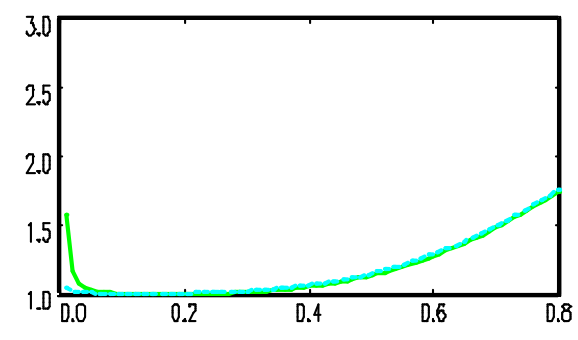

$\tau=-1 \quad \beta=1$
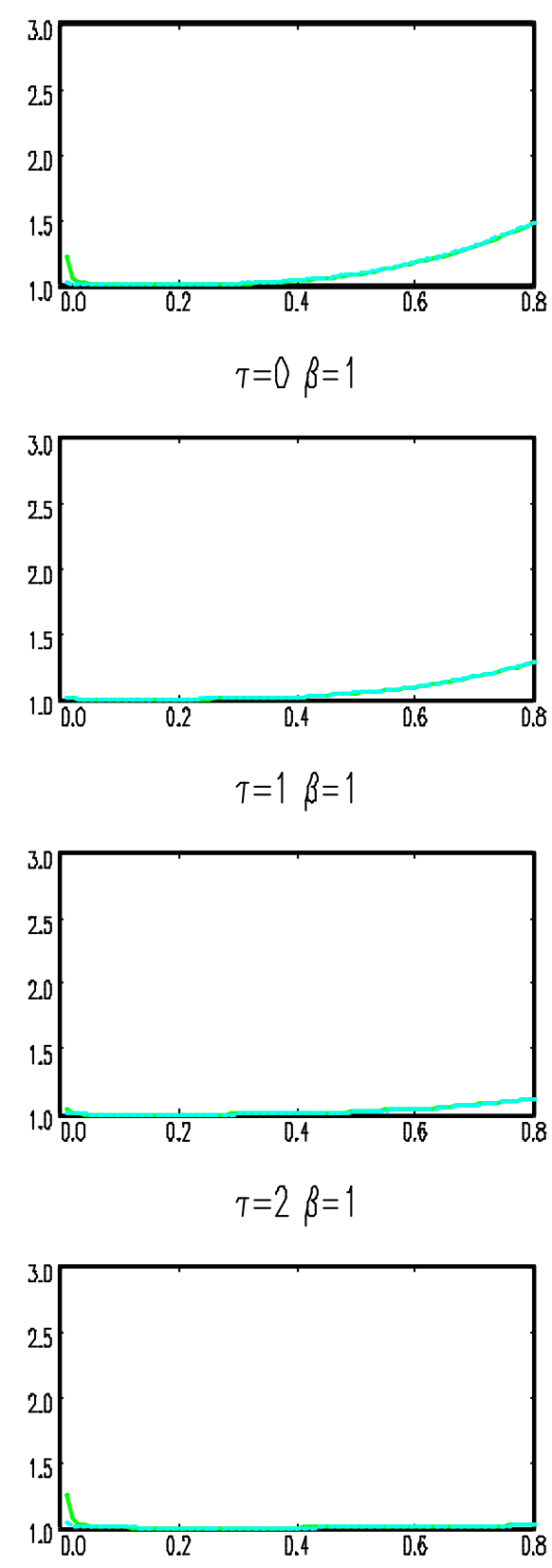

$T=-2 \beta=2$

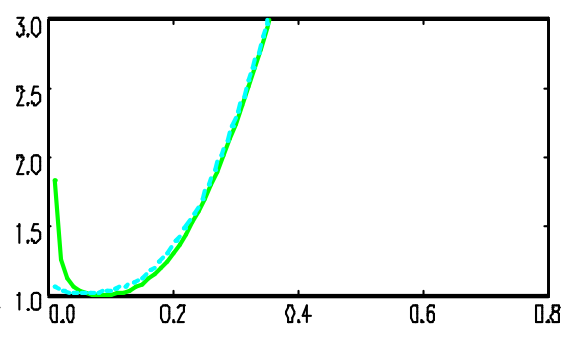

$\tau=-1 \quad \beta=2$

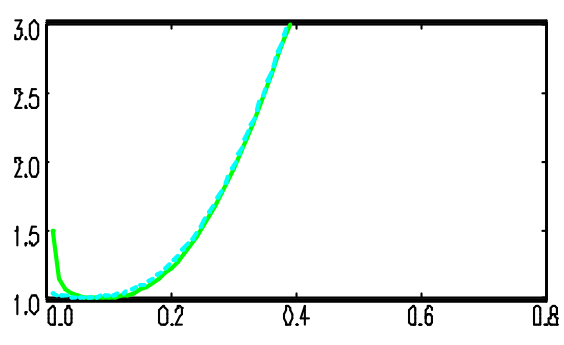

$\tau=0 \beta=2$

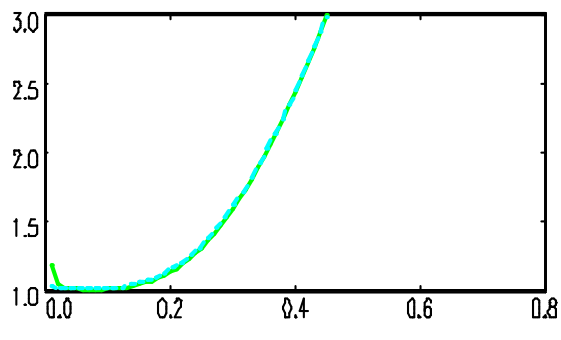

$\tau=1 \beta=2$

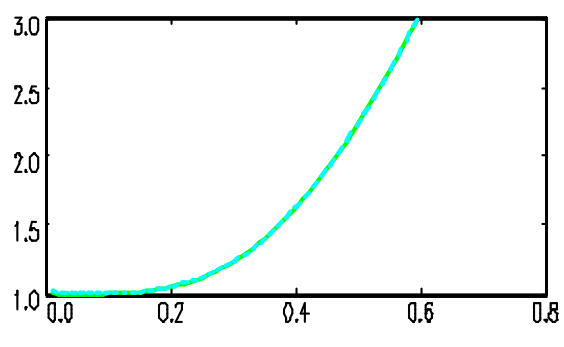

$\tau=2 \beta=2$

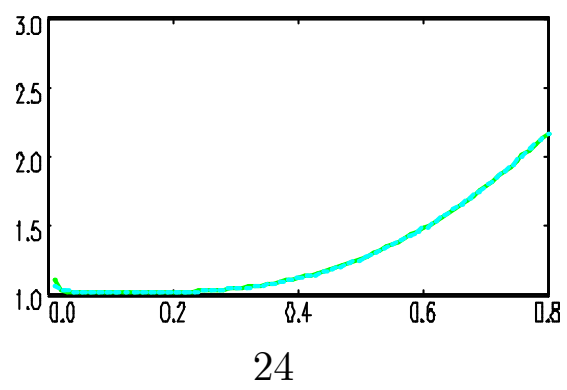

$T=-2 \beta=3$

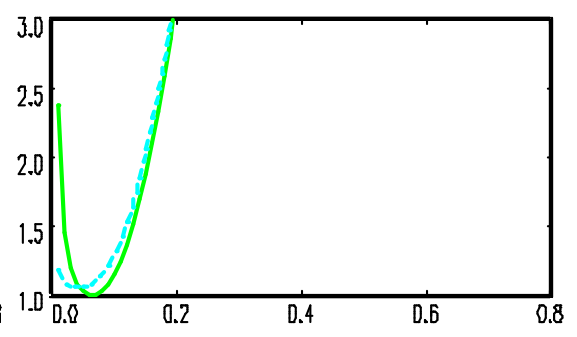

$\tau=-1 \beta=3$

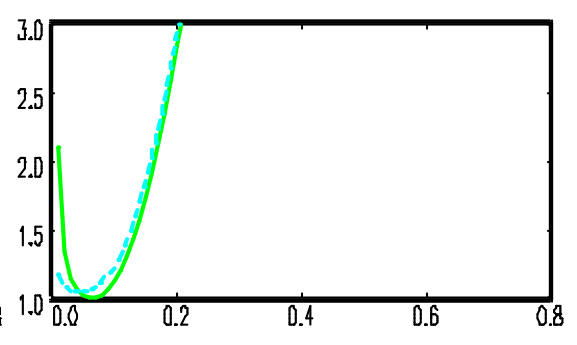

$\tau=0 \beta=3$

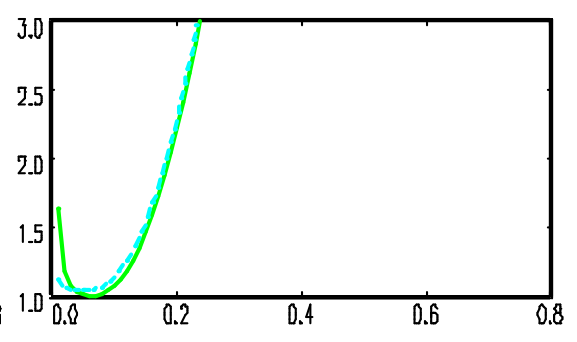

$\tau=1 \beta=3$

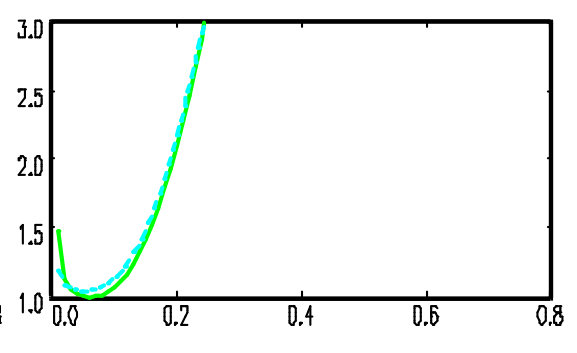

$\tau=2 \beta=3$

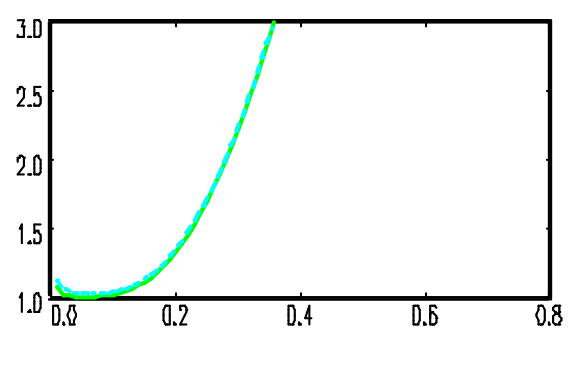

